\title{
Mutual inhibition between miR-34a and SIRT1 contributes to regulation of DNA double-strand break repair
}

\author{
XU Miao, LU Lu, MAO BeiBei, LÜ Xiang, WU XueSong, LI Lei* \& LIU DePei* \\ National Laboratory of Medical Molecular Biology, Institute of Basic Medical Sciences, Chinese Academy of Medical Sciences \& Peking Union \\ Medical College, Beijing 100005, China
}

Received September 17, 2012; accepted October 30, 2012; published online December 19, 2012

\begin{abstract}
DNA double-strand breaks are repaired through either non-homologous end joining (NHEJ) or homologous recombination repair (HRR) pathway. The well-characterized regulatory mechanisms of double-strand break repair (DSBR) are mainly found at the level of complicated repair protein interactions and modifications. Regulation of DSBR at the transcriptional level was also reported. In this study, we found that DSBR can be regulated by miR-34a at the post-transcriptional level. Specifically, miR-34a, which can be activated by DNA damages, represses DSBR activities by impairing both NHEJ and HRR pathways in cultured cells. The repression is mainly through targeting the critical DSBR promoting factor SIRT1, as ectopically expressed SIRT1 without 3'-UTR can rescue the inhibitory roles of miR-34a on DSBR. Further studies demonstrate that SIRT1 conversely represses miR-34a expression. Taken together, our data show that miR-34a is a new repressor of DSBR and the mutual inhibition between miR-34a and SIRT1 may contribute to regulation of DNA damage repair.
\end{abstract}

miR-34a, SIRT1, coregulation, DNA double-strand break repair, DNA damaging agent

Citation: Xu M, Lu L, Mao B B, et al. Mutual inhibition between miR-34a and SIRT1 contributes to regulation of DNA double-strand break repair. Chin Sci Bull, 2013, 58: 979-985, doi: 10.1007/s11434-012-5599-8

MicroRNAs (miRNAs) are posttranscriptional regulatory molecules that have been implicated in a wide variety of normal physiological processes and many human diseases $[1,2]$. These small non-coding RNAs ( 20-22 nt) exert functions by site-specific targeting of untranslated regions located at either end of mRNA molecules. MiRNAs have been shown to finely tune gene expression and exert failsafe functions. It has been proposed that positive and negative transcriptional coregulation of a miRNA and its targets is prevalent in the human and mouse genomes [3]. Such fine-tuning mechanisms may provide potential coordinated regulation and robustness of certain cellular functions in animal system [4].

DNA double-strand break repair (DSBR) is a complex multistep process under coordinated regulation, as dysregulation of DSBR underpins many human diseases and even predisposes individuals to tumorigenesis [5]. There are two

\footnotetext{
*Corresponding authors (email: liudp@pumc.edu.cn; lilei.pumc@ gmail.com)
}

main pathways to repair DSBs: non-homologous end joining (NHEJ) or homologous recombination repair (HRR) [6]. The process of DSBR encompasses gene-expression regulation at the transcriptional and post-translational levels [7-11]. The extensive roles of miRNA in diverse pathophysiological processes suggest an exquisite network may also exist for posttranscriptional controls over DSBR. One recent study showed that miR-24-mediated downregulation of H2AX suppresses DNA repair in terminally differentiated blood cells [12].

SIRT1 has recently been regarded as a critical player in DNA damage repair [13]. A study using DNA strand break repair reporter system in cell lines showed that ectopically expressed SIRT1 resulted in increased repair of strand breakage, while repression of endogenous SIRT1 by siRNA led to decrease of repair activity [14]. Other two recent studies using primary MEFs or ES cells also confirmed that SIRT1 can localize to break site and is required for efficient DSBR. Knock-out or depletion of SIRT1 resulted in im- 
paired nuclear foci formation of RAD51, NBS1, and greatly decreased repair activity $[15,16]$.

In this study, we observed that miR-34a represses plasmid re-ligation activity as well as repair of etoposide induced genomic DSBs. In addition, miR-34a inhibits homologous recombination (HR) mediated repair of I-SceI induced DSBs. Further rescue experiments showed that repression of miR-34a on DSBR is mediated by targeting SIRT1. Finally, SIRT1 was shown to inversely downregulate expression of miR-34a. These data indicate that the mutual inhibition between miR-34a and SIRT1 contributes to the regulation of DSBR.

\section{Materials and methods}

\subsection{Cell Culture}

HeLa, 293T, HEK-293 and MCF-7 cells were maintained in DMEM media (Invitrogen) supplemented with 10\% FBS. HEK-293A cells were maintained in $\alpha$-MEM media (Invitrogen) supplemented with $10 \%$ FBS.

\subsection{Plasmid construction}

Genomic regions containing pri-miR-34s and flanking sequences were PCR amplified from HeLa cell genome and inserted into pCR3.1-Uni to construct pCR3.1-34a expression plasmid. The promoter regions of miR-34a and miR34b/c loci were PCR amplified from HeLa cell genome and inserted into pGL3-basic to generate pGL3-34ap and pGL334bcp. The 3'-UTR fragments of SIRT1 and other repair factors were cloned into pGL3cM vector. The corresponding seed regions were then mutated using QuikChange method. For expression of miR-34a and SIRT1 through adenovirus, the corresponding genomic region of pri-miR34a or SIRT1 cDNA was cloned into pAd-Track-CMV and then shuttled into pAdEasy-1 followed by PacI digestion and transfection into HEK-293A cells [17]. The first generation viruses were harvested $7-8 \mathrm{~d}$ later.

\subsection{Luciferase assay}

HeLa, 293 T or HEK-293 cells were plated at $(0.5-1) \times 10^{4}$ per well in 24-well plates. On the next day, 100 ng pGL334ap plasmids were transfected into SIRT1-RNAi or Control-RNAi cells using Lipofectamine 2000 (Invitrogen). For 3'-UTR reporter assay, $100 \mathrm{ng}$ 3'-UTR plasmids were transfected along with $600 \mathrm{ng}$ miR-34a expression plasmids. For plasmid re-ligation assay, $100 \mathrm{ng}$ Eco RI-linearized pGL2-Luc plasmids or the circular pGL2-Luc plasmids were transfected along with $600 \mathrm{ng}$ miR-34a expression plasmids. Forty-eight hours later, cells were harvested and luciferase assays were performed by using Dual Luciferase Reporter Assay system (Promega).

\subsection{Neutral comet assay}

After adenovirus infection for $48 \mathrm{~h}$, HeLa cells were treated with $50 \mu \mathrm{mol}$ etoposide for $1 \mathrm{~h}$. Cells were then collected and processed according to the procedures of neutral comet assay that specifically detects DNA double strand breaks [18]. The experiment was repeated three times and the data were analyzed by using CometScore (TriTek).

\subsection{Cell cycle and apoptosis analysis}

The cells after different treatments were trypsinized, collected by centrifugation and fixed in $70 \%$ ethanol. After two washes in PBS, cells were treated with RNaseA and stained with propidium iodide for analysis by flow cytometry. SubG1 phase was measured to represent apoptotic cells.

\subsection{RNA interference}

The sense strand for shRNA of SIRT1 is 5'-GATGAAGTTGACCTCCTCA-3' [19], and the shRNA oligos were ligated into pXSN-mU6 [20]. The pXSN-shSIRT1 or pXSN-shGFP plasmids were trasnfected into GP293 cells along with pVSV-G to generate retroviruses. After infection, $600 \mu \mathrm{g} / \mathrm{mL}$ of G418 was used to generate stable knockdown cell lines.

\section{7 qRT-PCR and miRNA real-time PCR}

Total RNA was isolated by using Trizol (Invitrogen) reagent and reverse transcription was performed per the manufacturer's manual (New England Biolab). Real-time PCR was performed using SYBR Premix Ex Taq (TaKaRa) on Bio-Rad iQ5 cycler. Reverse transcription using stem-loop primers and real-time PCR of miR-34a and U6 RNA were performed as previously described [21]. The primer sequences are as follows:

$>$ RT primer for human U6 (RT-U6)

5'-GTCGTATCCAGTGCAGGGTCCGAGGTATTCGCACTGGATACGACAAAAATATG-3'

$>$ RT primer for hsa-miR-34a (RT-34a)

5'-GTCGTATCCAGTGCAGGGTCCGAGGTATTCGCACTGGATACGACAACAAC-3'.

Q-PCR-primer sequences are as follows:

>Forward primer for human u6 (qU6-F): 5'-GCGCGT-

CGTGAAGCGTTC-3'

$>$ Reverse primer for human u6 (qmiR-R): 5'-GTGCAGGGTCCGAGGT-3'

>Forward primer for hsa-miR-34a (q34a-F): 5'-GCGCTGGCAGTGTCTTAGC-3'

$>$ Reverse primer for hsa-miR-34a (qmiR-R): 5'-GTGCAGGGTCCGAGGT-3'.

\subsection{Chromatin immunoprecipitation (ChIP)}

ChIP experiments were performed as described by the Up- 
state protocol with some modifications. After crosslinking with formaldehyde, nuclei were isolated and lysed in SDS lysis buffer (1\% SDS, $10 \mathrm{mmol}$ EDTA and $50 \mathrm{mmpol}$ Tris, $\mathrm{pH}$ 8.1) and sonicated with Branson 150 sonicator to achieve fragment size of 200-750 bp. SIRT1 antibody (sc15404, Santa Cruz) was used for immunoprecipitation. The primers were used to amplify the promoter region of hsamiR-34a gene locus and the forward and reverse sequences are 5'-ACGGACTGAGAAACACAAGCG-3' and 5'-CTTCTCGGTGACCACGCAG-3' respectively. The primers for GAPDH promoter are 5'-TACTAGCGGTTTTACGGGCG-3' and 5'-TCGAACAGGAGGAGCAGAGAG-3' respectively.

\subsection{Immunoblotting and antibodies}

Cells were lysed with RIPA buffer (50 mmol Tris-HCl, $\mathrm{pH}$ $8,150 \mathrm{mmol} \mathrm{NaCl}, 1 \% \mathrm{NP}-40,0.5 \%$ sodium deoxycholate, $0.1 \%$ SDS, protease inhibitor cocktail from Sigma). Ku70 antibody (sc-1486) and SIRT1 antibody (sc-15404) were obtained from Santa Cruz Biotechnology, Inc. RAD51 antibody (PC130, Ab-1) was manufactured by Calbiochem. Anti- $\beta$-actin antibody was from Sigma.

\subsection{Statistical analysis}

Unpaired $t$-test analysis with two-tailed $P$-value was performed with the GraphPad Prism 5 software (GraphPad Software, San Diego, CA).

\section{Results}

\subsection{MiR-34a represses DSBR by impairing both NHEJ and HRR pathways}

MiR-34a has recently been shown to response to p53 and serve as a tumor suppressor [22-27]. Treatment of MCF-7 cells with low dose of DNA damaging agents, etoposide (VP16) or $\mathrm{H}_{2} \mathrm{O}_{2}$, apparently induces miR-34a expression (Figure S1), suggesting that miR-34a may involve in the regulation of DSBR.

NHEJ is one of the major processes for repairing DSBs, so we first determined whether miR-34a can affect DSBR through this pathway. To this end, we constructed expression vectors of human miR-34s and confirmed their expression by Northern blot (Figure 1(a)). Then we performed

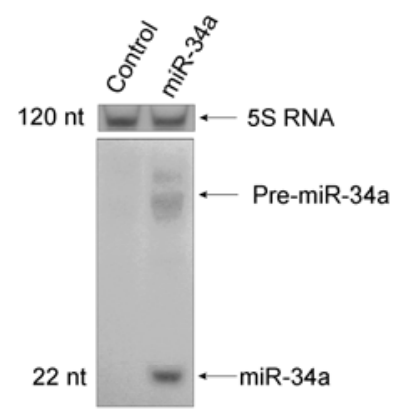

(a)

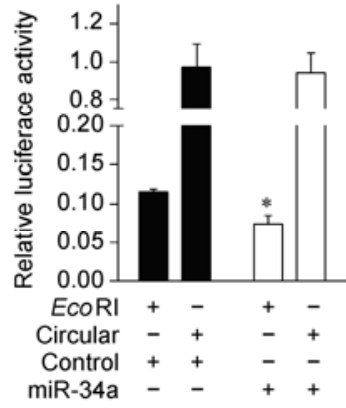

(b)
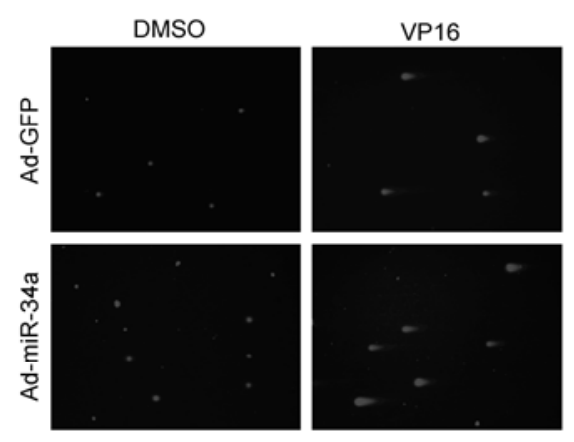

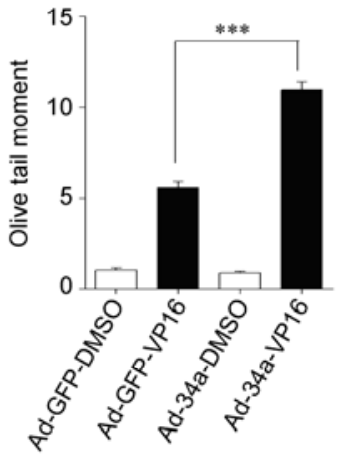

(c)

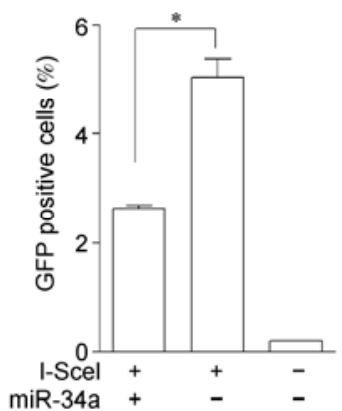

(d)

Figure 1 MiR-34a represses DSBR activity by affecting NHEJ and HRR pathways. (a) MiR-34a expression plasmids or control plasmids were transfected into HeLa cells, and $\gamma-{ }^{32} \mathrm{P}$ labeled anti-miR-34a oligo DNA probes (5'-ACAACCAGCTAAGACACTGCCA-3') were used to detect miR-34a expression. 5S RNA served as loading control. (b) MiR-34a expression plasmids were transfected into HeLa cells along with either circular or EcoRI-linearized pGL2-Luc plasmids and luciferase activity was measured $60 \mathrm{~h}$ post-transfection. Luciferase activity was normalized to Renilla Luciferase. Three independent experiments were performed and results were represented as mean \pm SD. (c) HeLa cells infected with Ad-GFP or Ad-miR-34a adenoviruses were treated with 50 $\mu \mathrm{mol} / \mathrm{L}$ etoposide or DMSO for $1 \mathrm{~h}$. Cells were then harvested for neutral comet assay. Representative pictures were shown here and the data were analyzed from three independent experiments ( $>100$ cells were analyzed). Olive tail moment was used to measure the amount of DSBs, and the data were shown as mean \pm SEM. Unpaired $t$-test was performed by GraphPad Prism and $* * *$ means $P<0.001$. (d) MiR-34a expression plasmids or control plasmids were transfected into DR-HEK-293 cells for $24 \mathrm{~h}$ followed by transfection with pI-SceI, and the cells were harvested $72 \mathrm{~h}$ after the first transfection for flow cytometry analysis. 
plasmid re-ligation assay using pGL2-Luc vectors that were linearized with Eco RI. Since Eco RI cuts pGL2-Luc at the coding region, precise re-ligation is needed for restoration of luciferase activity. Upon ectopic expression of miR-34a, the re-ligation efficiency of the damaged plasmids was reduced by about $20 \%$ (Figure 1(b)). The luciferase activities of control circular plasmids were not affected by the expression of miR-34a, which indicates specific inhibitory effects of miR-34a on ligation of damaged plasmids. These data demonstrate that miR-34a reduces the microhomologymediated precise end joining.

Then we examined whether miR-34a affects the repair of genomic DSBs caused by DNA damaging agents. To introduce DSBs, low dose of etoposide was adopted, and DSBR activity was measured by neutral comet assay. The results showed that ectopic expression of miR-34a led to about two-fold decrease of DSBR (increased comet tail moment) compared to the Ad-GFP group (Figure 1(c)).

Finally, we investigated whether miR-34a affects HRR pathway. The DR-GFP reporter system [28] was applied for this purpose. HEK-293 cells with stable integration of DR-GFP reporter were transfected with miR-34a expression plasmids or control plasmids followed by transfection with I-SceI-expressing plasmids $24 \mathrm{~h}$ later. Flow cytometry analysis showed that miR-34a reduced the efficiency of I-SceI induced HRR of mutated DR-GFP reporter by two folds (Figure 1(d)). Meanwhile, the vector only group was included to represent spontaneous recombination events (Figure 1(d)).

\subsection{Repression of DSBR by miR-34a is independent of the pro-apoptotic activity}

Overexpression of miR-34a has the potential to induce cell apoptosis, which may interfere with the detection of DNA repair. Therefore, we examined if miR-34a at the level we used for DNA repair assay affected cell growth. Ectopic expression of miR-34s in HeLa cells at the levels we used, however, did not change phase distribution of cell cycle or cause apoptosis (Figure 2(a) and (b)). These results showed that inhibition of DSBR activity caused by miR-34a in HeLa cells is independent of the pro-apoptotic effects.

\subsection{Inhibitory effects of miR-34a on DSBR is mediated by targeting SIRT1}

Since SIRT1 is a critical DSBR promoting factor that has also been identified as a miR-34a target in HCT116 and PC3 cells [29,30], it may be responsible for repression effects of miR-34a on DSBR. Immunoblot analysis showed that miR-34a decreased SIRT1 protein level significantly but did not apparently affect the expression of $\mathrm{Ku} 70$ and RAD51 (Figure 3(a)), which are also critical players for NHEJ and HRR respectively. SIRT1 mRNA level was also observed to be slightly decreased upon miR-34a expression
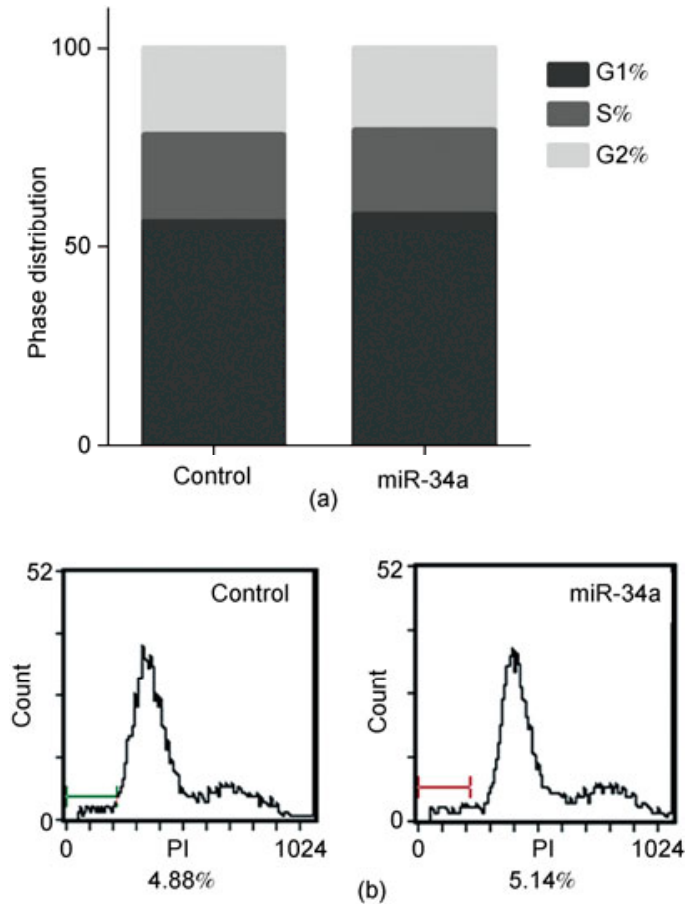

Figure 2 MiR-34a does not induce apoptosis in HeLa cells. HeLa cells transfected with miR-34a expression vectors were harvested $72 \mathrm{~h}$ posttransfection for flow cytometry analysis. The distribution of cell cycle phases was shown in the stacked bar graph (a) and the sub-G1 portion was also displayed (b).

(Figure 3(b)). Luciferase assay further confirmed that miR34a directly targeted wild type SIRT1 3 '-UTR but not the miR-34a binding-site-mutated form (Figure 3(c)).

To further prove that miR-34a represses DSBR by targeting SIRT1, we performed DNA repair assays by cotransfecting SIRT1 cDNA without 3'-UTR. Plasmid religation assay showed that upon introduction of SIRT1 cDNA, the ligation efficiency was significantly restored (Figure 3(d)). Neutral comet assay showed that the lowdose etoposide induced DSBs were repaired more efficiently in SIRT1 expression group (decreased comet tail moment) compared with the miR-34a only group (Figure 3(e)). DRGFP reporter assay by flow cytometry also showed that exogenous SIRT1 expression, as expected, can partially rescue the inhibitory effect of miR-34a on HRR (Figure 3(f)). Taken together, it was concluded that miR-34a represses DSBR activities mainly by targeting SIRT1.

\subsection{SIRT1 inversely represses expression of miR-34a}

MicroRNAs often co-regulate with their targets to realize fine-tuning of certain cellular functions [3], so it is intriguing to test if SIRT1 can inversely regulate expression of miR-34a. First, the specific inhibitor of SIRT1, Sirtinol was used to treat HEK-293 cells and miR-34a expression was observed to be increased (Figure 4(a)). Second, ectopic expression of dominant-negative SIRT1 (H363Y) form, which lacks catalytic deacetylase activity caused by H-to-Y 


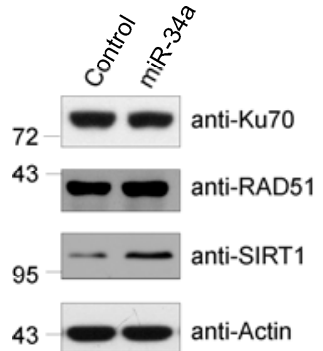

(a)

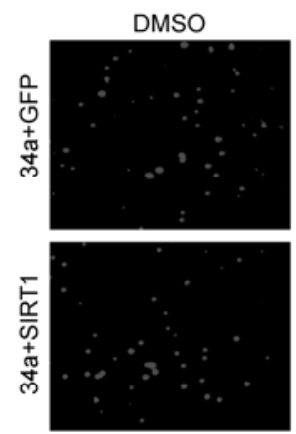

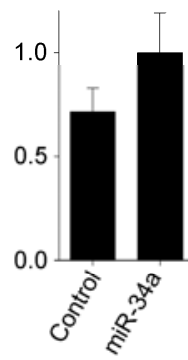

(b)

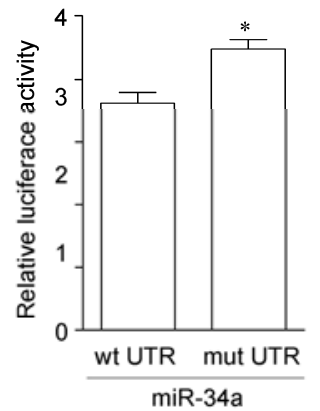

(c)

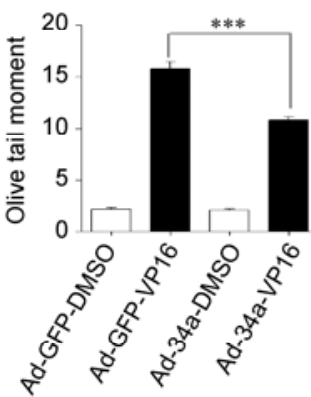

(e)

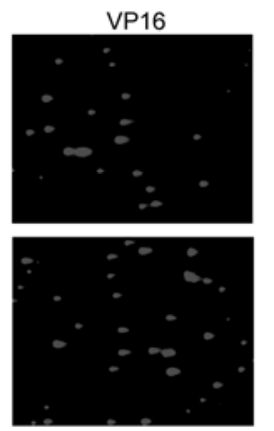

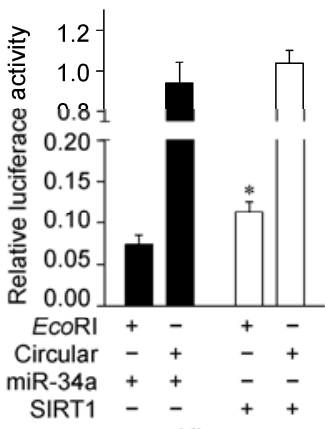

(d)

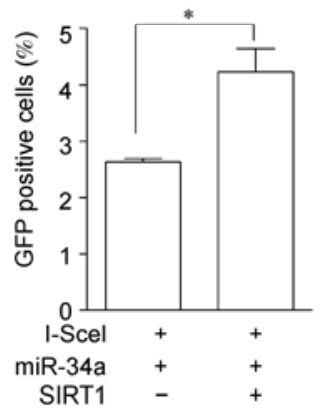

(f)

Figure 3 Repression of miR-34a on DSBR is mediated by targeting SIRT1. (a) HeLa cells infected with Ad-miR-34a or control Ad-GFP viruses were harvested $72 \mathrm{~h}$ post infection and Western blot was performed to detect Ku70, RAD51 and SIRT1 expression, and $\beta$-actin served as loading control. (b) Samples indicated in (a) were analyzed for SIRT1 mRNA level by qRT-PCR and results were shown as mean \pm SD. Expression level was normalized to GAPDH. (c) The reporter plasmids containing wild-type (wt UTR) or miR-34-site-mutated (mut UTR) fragments of SIRT1 3'-UTR were transfected into HeLa cells along with miR-34a expression plasmids. Relative luciferase activities were measured and shown as mean \pm SD. (d) MiR-34a and SIRT1 (without 3'-UTR) expression plasmids were co-transfected into HeLa cells along with either circular or EcoRI-linearized pGL2-Luc plasmids. The data were analyzed as in Figure 1(b). (e) HeLa cells co-infected with Ad-GFP and Ad-miR-34a, or Ad-SIRT1 (without 3'-UTR) and Ad-miR-34a adenoviruses were treated with $50 \mu \mathrm{mol} / \mathrm{L}$ etoposide or DMSO for $1 \mathrm{~h}$. Neutral comet assay was performed and analyzed as in Figure 1(c). (f) SIRT1 (without 3'-UTR) and miR-34a expression plasmids were co-transfected into DR-HEK-293 cells for $24 \mathrm{~h}$ followed by transfection with pI-SceI, and the flow cytometry analysis was performed as in Figure 1(d).

mutation at position 363 of human SIRT1 protein, also caused up-regulation of miR-34a (Figure 4(b)). Subsequently, the promoter activity of miR-34a was evaluated in SIRT1-RNAi cells (Figure 4(c) and (d)). The results showed that miR-34a promoter activity increased in SIRT1-RNAi cells (Figure 4(e)). ChIP experiment also detected that SIRT1 can be recruited to the promoter region of miR-34a gene (Figure 4(f)). RT-PCR analysis further showed that endogenous miR-34a expression was up-regulated upon SIRT1 knockdown (Figure 4(g)). Therefore SIRT1 negatively regulates miR-34a expression, and the two forms negative co-regulation relationship, which potentially regulates DSBR activity.

\section{Discussion}

MiRNAs participate in diverse biological processes in plants and animals, and it is possible that positive and negative coregulation of miRNA and its targets widely exist [3]. Such characteristics may reflect functional diversity and fine-tuning regulation by miRNAs. The extensive roles of
miRNA suggest that an exquisite network may contribute to posttranscriptional regulation of DSBR.

In this study, we have verified that miR-34a has inhibitory roles on DSBR. As for the NHEJ pathway, decreased repair efficiency of EcoRI-linearized plasmid DNA (pGL2Luc) and etoposide induced genomic DSBs were detected upon ectopic expression of miR-34a (Figure 1(b) and (c)). As the central player of NHEJ pathway, Ku70 expression was detected by immunoblot and no apparent changes were observed (Figure 3(a)). The 3'-UTR of Ku70 was also scanned by TargetScan [31] and no potential miR-34s binding sites were found. We reasoned that the impaired genomic DSBR is ascribed to inhibition of SIRT1 by miR-34a, and this was then supported by the rescue experiment in which exogenous SIRT1 cDNA without 3'-UTR was introduced (Figure 3(e)). In addition, miR-34a also suppresses HRR of DSBs induced by I-SceI at the chromosomal DR-GFP loci. Although miR-34a has putative targeting site at 3'-UTR of RAD51 (Figure S2), it has very mild effects on protein level of RAD51 (Figure 3(a)). Thus, the inhibitory effect of miR-34a on HRR is independent of RAD51. Introduction of SIRT1 cDNA without 3'-UTR, 


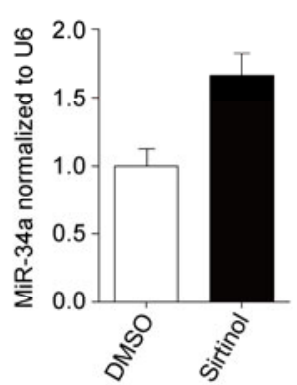

(a)

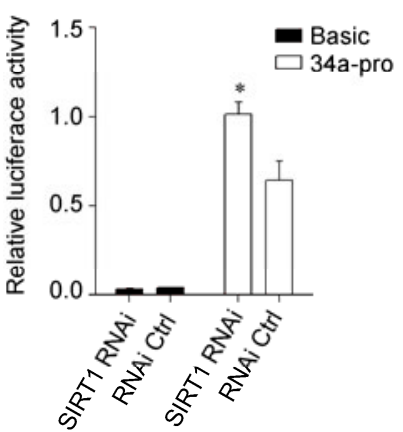

(e)

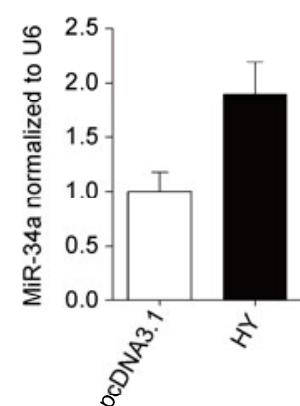

(b)

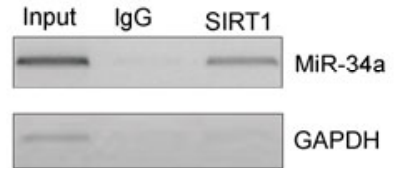

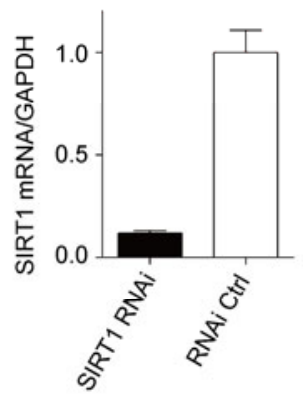

(c)

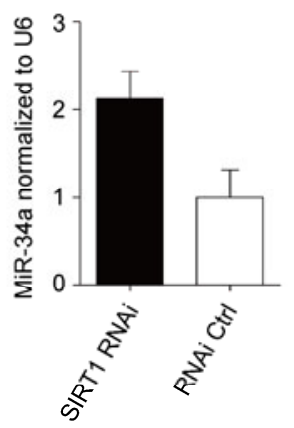

(g)

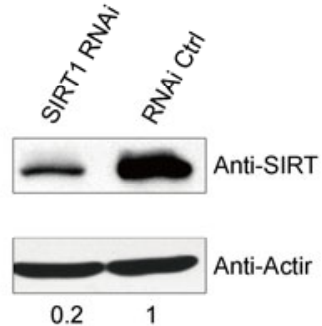

(d)

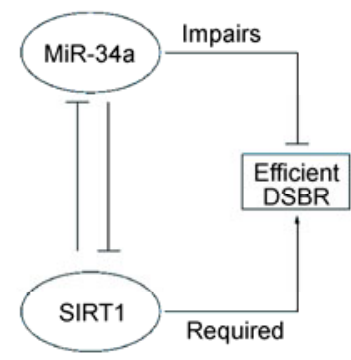

(h)

Figure 4 SIRT1 down-regulates MiR-34a expression. (a) and (b) HEK-293 cells were treated with $30 \mu \mathrm{mol} / \mathrm{L}$ Sirtinol or transfected with dominant-negative SIRT1 (HY), and MiR-34a expression was detected by qRT-PCR and normalized to U6 snRNA. (c) SIRT1 mRNA level was detected in SIRT1-RNAi cells and Control-RNAi cells, and normalized to GAPDH. (d) SIRT1 protein level was analyzed by immunoblot assay and $\beta$-Actin served as loading control. (e) MiR-34a promoter reporter plasmids or pGL3-basic plasmids were transfected into SIRT1-RNAi cells or control cells and luciferase activities were measured. Results are represented as mean \pm SD. (f) ChIP experiment was performed using SIRT1 antibody or normal IgG, and miR-34a promoter region was amplified by PCR. The amplified promoter region of MiR-34a contains p53 binding site. GAPDH promoter served as negative control. (h) Schematic presentation was shown to depict mutual inhibition between MiR-34a and SIRT1, and their roles in regulating DSBR. (g) MiR-34a expression level in SIRT1-RNAi or control cells was analyzed by qRT-PCR as described in (a).

however, can partially rescue the inhibitory effect by miR$34 \mathrm{a}$, suggesting that miR-34a suppresses HRR by targeting SIRT1 (Figure 3(f)). A recent study by Uhl et al. [32] also proved that SIRT1 activity promotes HRR in human cells, and this effect is independent of RAD51 but depends on Werner helicase. Therefore, miR-34a regulates DSBR at the upstream level by affecting SIRT1 expression.

SIRT1 was shown here to downregulate miR-34a by being recruited to the promoter region (Figure $4(\mathrm{~g}))$. This makes it possible that SIRT1 and p53 counteract in regulating miR-34a expression in response to DNA damages. Upon DSBs formation, p53 is activated by phosphorylation and then functions as a "molecular node" in the DNAdamage response. p53 represses HRR via direct interaction with and inhibition of RAD51 and this is independent of p53 transactivation function [33,34]. Activated wild-type p53 can also suppress NHEJ although such a function and underlying mechanisms still bear debates [35]. In this study we found that p53-transactivated miR-34a inhibits DSBR by targeting SIRT1 at the posttranscriptional level (Figure 3 ). The inhibition of DSBR by miR-34a adds new contents to functional network of p53. In summary, the activation of miR-34a by DSBs and repression by SIRT1 may contribute to modulating DNA repair activity and balancing cellular responses to different genotoxic stress (Figure 4(h)).

This work was supported by the National Basic Research Program of China (2011CB965203) and the National Natural Science Foundation of China (31030026 and 31021091).

1 Filipowicz W, Bhattacharyya S N, Sonenberg N. Mechanisms of post-transcriptional regulation by microRNAs: Are the answers in sight? Nat Rev Genet, 2008, 9: 102-114

2 Inui M, Martello G, Piccolo S. MicroRNA control of signal transduction. Nat Rev Mol Cell Biol, 2010, 11: 252-263

3 Tsang J, Zhu J, van Oudenaarden A. MicroRNA-mediated feedback and feedforward loops are recurrent network motifs in mammals. Mol Cell, 2007, 26: 753-767

4 Ebert M S, Sharp P A. Roles for microRNAs in conferring robustness to biological processes. Cell, 2012, 149: 515-524

5 McKinnon P J, Caldecott K W. DNA strand break repair and human genetic disease. Annu Rev Genomics Hum Genet, 2007, 8: 37-55

6 O'Driscoll M, Jeggo P A. The role of double-strand break repairInsights from human genetics. Nat Rev Genet, 2006, 7: 45-54

7 Huen M S, Chen J. The DNA damage response pathways: At the crossroad of protein modifications. Cell Res, 2008, 18: 8-16

8 Arias-Lopez C, Lazaro-Trueba I, Kerr P, et al. p53 modulates homologous recombination by transcriptional regulation of the RAD51 gene. EMBO Rep, 2006, 7: 219-224 
9 Bindra R S, Glazer P M. Repression of RAD51 gene expression by E2F4/p130 complexes in hypoxia. Oncogene, 2007, 26: 20482057

10 Wakasugi T, Izumi H, Uchiumi T, et al. ZNF143 interacts with p73 and is involved in cisplatin resistance through the transcriptional regulation of DNA repair genes. Oncogene, 2007, 26: 5194-5203

11 Mullan P B, Quinn J E, Harkin D P. The role of BRCA1 in transcriptional regulation and cell cycle control. Oncogene, 2006, 25: 5854-5863

12 Lal A, Pan Y, Navarro F, et al. MiR-24-mediated downregulation of H2AX suppresses DNA repair in terminally differentiated blood cells. Nat Struct Mol Biol, 2009, 16: 492-498

13 Gorospe M, de Cabo R. AsSIRTing the DNA damage response. Trends Cell Biol, 2008, 18: 77-83

14 Jeong J, Juhn K, Lee H, et al. SIRT1 promotes DNA repair activity and deacetylation of Ku70. Exp Mol Med, 2007, 39: 8-13

15 Wang R H, Sengupta K, Li C, et al. Impaired DNA damage response, genome instability, and tumorigenesis in SIRT1 mutant mice. Cancer Cell, 2008, 14: 312-323

16 Oberdoerffer P, Michan S, McVay M, et al. SIRT1 redistribution on chromatin promotes genomic stability but alters gene expression during aging. Cell, 2008, 135: 907-918

17 Luo J, Deng Z L, Luo X, et al. A protocol for rapid generation of recombinant adenoviruses using the AdEasy system. Nat Protoc, 2007, 2: $1236-1247$

18 Olive P L, Banath J P. The comet assay: A method to measure DNA damage in individual cells. Nat Protoc, 2006, 1: 23-29

19 Picard F, Kurtev M, Chung N, et al. SIRT1 promotes fat mobilization in white adipocytes by repressing PPAR-gamma. Nature, 2004, 429: 771-776

20 Liu C M, Liu D P, Dong W J, et al. Retrovirus vector-mediated stable gene silencing in human cell. Biochem Biophys Res Commun, 2004, 313: 716-720

21 Chen C, Ridzon D A, Broomer A J, et al. Real-time quantification of microRNAs by stem-loop RT-PCR. Nucleic Acids Res, 2005, 33: e179

22 Bommer G T, Gerin I, Feng Y, et al. p53-mediated activation of miRNA34 candidate tumor-suppressor genes. Curr Biol, 2007, 17:
1298-1307

23 He L, He X, Lim L P, et al. A microRNA component of the p53 tumour suppressor network. Nature, 2007, 447: 1130-1134

24 Welch C, Chen Y, Stallings R L. MicroRNA-34a functions as a potential tumor suppressor by inducing apoptosis in neuroblastoma cells. Oncogene, 2007, 26: 5017-5022

25 Chang T C, Wentzel EA, Kent O A, et al. Transactivation of miR-34a by p53 broadly influences gene expression and promotes apoptosis. Mol Cell, 2007, 26: 745-752

26 Raver-Shapira N, Marciano E, Meiri E, et al. Transcriptional activation of miR-34a contributes to p53-mediated apoptosis. Mol Cell, 2007, 26: 731-743

27 Tazawa H, Tsuchiya N, Izumiya M, et al. Tumor-suppressive miR34a induces senescence-like growth arrest through modulation of the E2F pathway in human colon cancer cells. Proc Natl Acad Sci USA, 2007, 104: 15472-15477

28 Pierce A J, Johnson R D, Thompson L H, et al. XRCC3 promotes homology-directed repair of DNA damage in mammalian cells. Genes Dev, 1999, 13: 2633-2638

29 Fujita Y, Kojima K, Hamada N, et al. Effects of miR-34a on cell growth and chemoresistance in prostate cancer PC3 cells. Biochem Biophys Res Commun, 2008, 377: 114-119

30 Yamakuchi M, Ferlito M, Lowenstein C J. MiR-34a repression of SIRT1 regulates apoptosis. Proc Natl Acad Sci USA, 2008, 105: 13421-13426

31 Lewis B P, Burge C B, Bartel D P. Conserved seed pairing, often flanked by adenosines, indicates that thousands of human genes are microRNA targets. Cell, 2005, 120: 15-20

32 Uhl M, Csernok A, Aydin S, et al. Role of SIRT1 in homologous recombination. DNA Repair (Amst), 2010, 9: 383-393

33 Dudenhoffer C, Kurth M, Janus F, et al. Dissociation of the recombination control and the sequence-specific transactivation function of p53. Oncogene, 1999, 18: 5773-5784

34 Willers H, McCarthy E E, Wu B, et al. Dissociation of p53-mediated suppression of homologous recombination from G1/S cell cycle checkpoint control. Oncogene, 2000, 19: 632-639

35 Sengupta S, Harris C C. P53: Traffic cop at the crossroads of DNA repair and recombination. Nat Rev Mol Cell Biol, 2005, 6: 44-55

Open Access This article is distributed under the terms of the Creative Commons Attribution License which permits any use, distribution, and reproduction in any medium, provided the original author(s) and source are credited.

\section{Supporting Information}

Figure S1 Low-dose etoposide (VP16) and $\mathrm{H}_{2} \mathrm{O}_{2}$ induce miR-34a expression in MCF-7 cells.

Figure S2 MiR-34a potentially targets RAD51 3'-UTR. MiR-34s used in these assays were expressed from pMSCV-IRES-Puro (PIG) constructs.

The supporting information is available online at csb.scichina.com and www.springerlink.com. The supporting materials are published as submitted, without typesetting or editing. The responsibility for scientific accuracy and content remains entirely with the authors. 